\title{
Predictors of Survival in Favorable Risk Patients with Metastatic Renal Cell Carcinoma Treated with a Single-Agent First-Line Therapy
}

\author{
Ryuichi Mizuno $^{a}$ Kimiharu Takamatsu ${ }^{a}$ Yota Yasumizu ${ }^{a} \quad$ Nobuyuki Tanaka ${ }^{a}$ \\ Toshikazu Takeda $^{a}$ Shinya Morita ${ }^{a}$ Kazuhiro Matsumoto ${ }^{a}$ Takeo Kosaka $^{a}$ \\ Hiroshi Asanuma ${ }^{a}$ Shuji Mikamib Mototsugu Oya ${ }^{a}$ \\ ${ }^{a}$ Department of Urology, Keio University School of Medicine, Tokyo, Japan; bDivision of Diagnostic Pathology, Keio \\ University School of Medicine, Tokyo, Japan
}

\section{Keywords}

Kidney cancer · Favorable risk · International metastatic renal cell carcinoma database consortium

\begin{abstract}
Introduction: The aim of this retrospective study was to elucidate predictors of survival in metastatic renal cell carcinoma (mRCC) patients in an International Metastatic Renal Cell Carcinoma Database Consortium favorable risk group treated with frontline therapy without immune checkpoint inhibitors. Methods: A total of 238 patients with mRCC were reviewed. Among them, 55 patients in favorable risk group treated with single-agent systemic therapy were retrospectively analyzed. Clinical and pathological data were retrieved and analyzed retrospectively. The prognostic effect of each marker on overall survival (OS) was investigated with univariate and multivariate Cox's proportional hazards regression models. Results: After a median follow-up of 46.2 months after first-line treatment initiation, the median progression-free survival (PFS) was 29.3 months, and the median OS has not been reached. The estimated percentage of patients who were alive at 12 and 24 months were 96.1 and $94.1 \%$, respectively. Multivariate analysis revealed that the long-term duration of first-line treatment (hazard ratio [HR]:
\end{abstract}

$0.972,95 \%$ confidence interval [Cl]: 0.944-0.997, $p=0.0299$ ) and the metastases limited to lung (HR: 3.852, 95\% Cl: $1.080-$ 24.502, $p=0.0361$ ) were independent predictors for longer OS in favorable risk mRCC patients. Conclusion: First-line systemic therapy for favorable risk $\mathrm{mRCC}$ patients with a single agent resulted in relatively longer PFS and OS. A longer duration of first-line treatment and lung only metastases are correlated with longer OS.

(C) 2022 The Author(s)

Published by S. Karger AG, Basel

\section{Introduction}

With the development of effective molecular targeted drugs and immune checkpoint inhibitors (ICIs), much progress has been made in the treatment of advanced or metastatic renal cell carcinoma (mRCC) and survival has been prolonged. An improved understanding of the functional loss of the von Hippel Lindau tumor suppressor gene, which contributes to clear cell carcinoma initiation and tumor angiogenesis, has led to the development of tyrosine kinase inhibitors (TKIs) targeting mainly vascular endothelial growth factor receptors (VEGFR). Sunitinib and pazopanib are VEGFR-TKIs which block several pathways implicated in tumor growth and angiogenesis. Each agent has karger@karger.com www.karger.com/uin

Karger $\stackrel{\text { ' }}{5}$

GOPEN ACCESS
(C) 2022 The Author(s)

Published by S. Karger AG, Basel

This is an Open Access article licensed under the Creative Commons Attribution-NonCommercial-4.0 International License (CC BY-NC) (http://www.karger.com/Services/OpenAccessLicense), applicable to the online version of the article only. Usage and distribution for commercial purposes requires written permission.
Correspondence to:

Ryuichi Mizuno, mizunor@z7.keio.jp 
been shown to improve disease control in randomized clinical trials in frontline settings $[1,2]$. In recent years, immunotherapy using ICIs, targeting the programmed death-1/ programmed death-ligand 1 axis to drive antitumor immune responses have also been incorporated into the firstline treatment of mRCC. More recently, combination regimens, which include ICIs, have been replacing TKI monotherapy in the first-line treatment for mRCC [3-5]. Today, a spectrum of single-agent TKI to TKI/ICI and ICI/ICI combinations can be considered and decisions about the best regimen are complex.

In clinical practice, treatment decisions are frequently made based on risk stratification, such as the International Metastatic Renal Cell Carcinoma Database Consortium (IMDC) model. Among these risk groups, IMDC favorable risk patients have demonstrated much longer survivals even before the approval of ICIs for first-line treatment [6]. Several phase 3 studies comparing ICI combination therapies with a single agent have demonstrated that combination regimens improved progression-free survival (PFS) even in favorable risk patients $[4,5]$. However, overall survival (OS) outcomes were immature as of the primary analysis, especially in favorable risk patients. Based on those results, the National Comprehensive Cancer Network treatment guideline recommends some ICI regimens and single TKI treatment equally as preferred regimens in favorable risk patients (https://www.nccn.org/). Thus, there exist no indices with which to make first-line treatment decisions in favorable risk patients, especially for the use of ICIs. In addressing preliminary issues surrounding frontline treatment for patients in favorable risk groups, the elucidation of first-line treatment efficacy would help make treatment decisions. The main purpose of this study was to evaluate the correlation between clinical features and subsequent outcome of mRCC patients in an IMDC favorable risk group treated with frontline therapy without ICIs.

\section{Materials and Methods}

A total of 238 patients with mRCC, who were treated with systemic therapy at Keio University Hospital between 2008 and 2020, were reviewed. Among them, 60 cases were categorized into the IMDC favorable risk group. Five cases who received IO containing first-line regimen were excluded. Therefore, 55 cases in the IMDC favorable risk group were analyzed in this study. Clinical and pathological data from medical records including age, gender, tumor pathology, location of metastatic sites, type of first-line systemic therapy, baseline serum lactate dehydrogenase level, baseline Creactive protein (CRP) level, duration of first-line systemic therapy, and baseline body mass index were retrieved and analyzed retrospectively. All patients were assessed by medical examination
Table 1. Clinicopathological characteristics of 55 patients in favorable risk group

IMDC favorable risk $(n=55)(\%)$

\begin{tabular}{ll}
\hline Median age, years (range) & $64(30-81)$ \\
Gender male/female & $47(85.5) / 8(14.5)$ \\
Pathology clear/non clear & $51(92.7) / 4(7.3)$ \\
Location of metastatic sites, $n(\%)$ & \\
$\quad$ Lung & $27(49.0)$ \\
$\quad$ Lymph nodes & $17(30.9)$ \\
Adrenal glands & $6(10.9)$ \\
Bone & $5(9.1)$ \\
Pancreas & $5(9.1)$ \\
Liver & $4(7.3)$ \\
Other & $17(30.9)$ \\
Prior nephrectomy & $55(100.0)$ \\
First-line systemic therapy, $n(\%)$ & \\
$\quad$ TKI & $45(81.8)$ \\
mTOR inhibitor & $5(9.1)$ \\
Cytokines & $5(9.1)$
\end{tabular}

mTOR, mammalian target of rapamycin; IMDC: International Metastatic RCC Database Consortium.

and a blood test every $2-4$ weeks during systemic therapies. We conducted computed tomography for radiographic evaluation every 3-6 months. The radiographic response to systemic therapy was assessed using response evaluation criteria in solid tumors, version 1.1 [7]. Patients were stratified into 4 groups as follows: those with complete remission or partial response (PR), stable disease (SD), and progressive disease (PD). All procedures performed in studies involving human participants were in accordance with the ethical standards of the Keio University Research Ethics Committee (Approval No. 20130424) and with the 1964 Helsinki Declaration and its later amendments or comparable ethical standards.

PFS was defined as the time that had elapsed between the initiation of first-line systemic therapy and tumor progression. OS was defined as the time from initiation of first-line systemic therapy to death from any cause. PFS and OS were determined using the Kaplan-Meier method and analyzed using the log-rank test. The prognostic effect of each marker on OS was investigated with Cox's proportional hazards regression model. Univariate and multivariate analyses were performed to identify independent prognostic factors for OS. Statistical analysis was performed by JMP version 15.0.0 (SAS Institute, Inc., Cary, NC, USA) and $p$ values less than 0.05 were judged as statistically significant. We also assessed treatment-related adverse events by using the Common Terminology Criteria for Adverse Events version 4.0 in this cohort.

\section{Results}

The clinical and pathologic features of this cohort are summarized in Table 1. All patients had prior nephrectomy. Pathological diagnoses were clear cell carcinoma
Urol Int 2022;106:1145-1149 DOI: $10.1159 / 000521960$
Mizuno et al. 


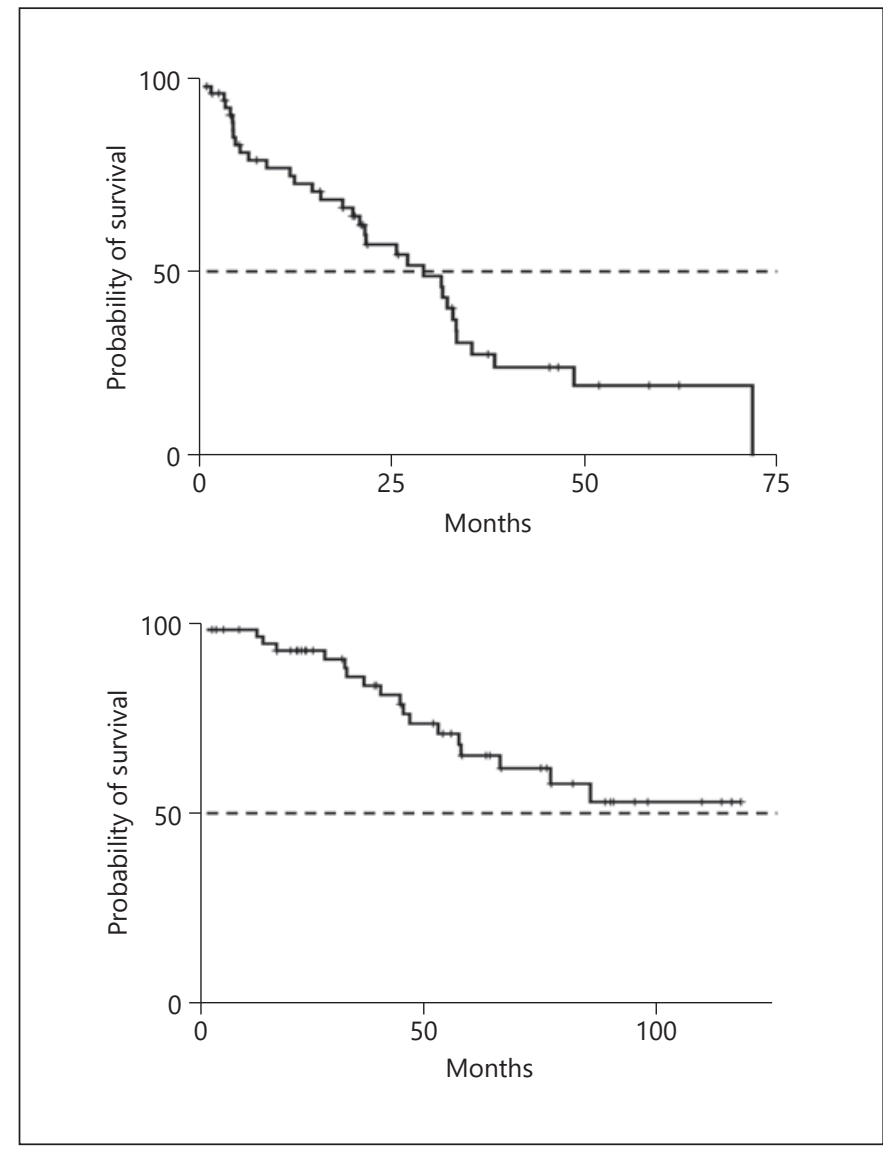

Fig. 1. Kaplan-Meier curves of PFS for first-line systemic therapy (a) and OS from first-line systemic therapy initiation of metastatic renal cell carcinoma patients in favorable risk group (b). + censored case.

$(51 / 55 ; 92.8 \%)$, papillary $(1 / 55 ; 1.8 \%)$, chromophobe $(1 / 55$; $1.8 \%), \mathrm{Xp} 11.2$ translocation $(1 / 55 ; 1.8 \%)$, and unclassified $(1 / 55 ; 1.8 \%)$, respectively. In total, $5(9.1 \%), 45(81.8 \%)$, and 5 (9.1\%), patients received cytokine, vascular endothelial growth factor-targeted, and mammalian target of rapamycin-targeted therapy as a first-line treatment, respectively. Four patients (7.3\%) achieved complete remission, 4 (7.3\%) had PR, $40(72.7 \%)$ had SD, and 5 (9.1\%) had PD. At the time of analysis, 43 patients (78.2\%) had discontinued their first-line therapies and 17 patients (30.9\%) had died. After a median follow-up of 46.2 months after treatment initiation, the median PFS was 29.3 months (95\% confidence interval [CI]: 20.8-33.6), and the median OS had not been reached (95\% CI: 56.8 - not reached) (Fig. 1). The estimated percentages of patients who were alive at 12 and 24 months were 96.1 and $94.1 \%$, respectively.

We next evaluated some clinical indices that could predict OS. In univariate Cox proportional hazards model
Table 2. Univariate and multivariate cox regression analyses for OS of patients in favorable risk group

\begin{tabular}{lllll}
\hline & $\begin{array}{l}\text { Univariate } \\
p \text { value }\end{array}$ & $p$ value & $\begin{array}{l}\text { Multivariate } \\
\mathrm{HR}\end{array}$ & $95 \% \mathrm{Cl}$ \\
\hline Age & 0.8804 & & & \\
Gender & 0.8989 & & & \\
Pathology & 0.9825 & & & \\
LDH & 0.4488 & & & \\
CRP & 0.1826 & & & \\
Metastatic sites & 0.0355 & 0.0361 & & \\
$\quad$ Exclusive lung & & & $1.000-24.502$ \\
$\quad$ Others & & & 3.852 & \\
$\begin{array}{l}\text { BMl } \\
\text { Duration of first-line }\end{array} \quad 0.2820$ & & & $0.944-0.997$ \\
$\quad$ systemic therapy & 0.0294 & 0.0299 & 0.972 & \\
\hline
\end{tabular}

$\mathrm{LDH}$, lactate dehydrogenase; CRP, C-reactive protein BMI, body mass index; HR: hazard ratio $\mathrm{Cl}$ : confidence interval

Table 3. Summary of adverse events during first-line systemic therapy

\begin{tabular}{lll}
\hline Adverse events & $N=55$ & \\
\cline { 2 - 3 } & All grades & Grade 3/4 \\
\hline Hypertension & 33 & 12 \\
Hand foot syndrome & 32 & 1 \\
Hypothyroidism & 17 & 0 \\
Diarrhea & 9 & 0 \\
Fever & 8 & 0 \\
Proteinuria & 7 & 1 \\
Liver dysfunction & 5 & 0 \\
Stomatitis & 5 & 0 \\
Acute kidney injury & 3 & 2 \\
Anemia & 3 & 0 \\
Appetite loss & 3 & 0 \\
Hemorrhage & 2 & 2 \\
Stroke & 2 & 1 \\
Hyperthyroidism & 1 & 0 \\
Hyperlipidemia & 1 & 0 \\
\hline
\end{tabular}

analyses, the long-term duration of first-line treatment $(p=$ $0.0294)$ and the metastases limited to the lung $(p=0.0355)$ were significantly correlated with prolonged OS (Table 2). No correlations were found between OS and age, gender, pathology, baseline lactate dehydrogenase, baseline CRP, and body mass index. Multivariate analysis revealed the long-term duration of first-line treatment (hazard ratio: 0.972, 95\% CI: 0.944-0.997, $p=0.0299$ ) and the metastases limited to the lung (hazard ratio: 3.852, 95\% CI: 1.080 $24.502, p=0.0361$ ) were independent predictors for longer OS in the favorable risk mRCC patients (Table 2). 
The rates of adverse events are summarized in Table 3 . Among those who discontinued first-line treatment due to toxicities $(N=8 ; 14.5 \%)$, the only toxicities leading to discontinuation occurring in more than 1 patient were renal dysfunction $(N=3)$, hemorrhage $(N=2)$, hyperthyroidism, stroke, and liver function test abnormalities $(N=1$ each). Twelve out of 55 (21.8\%) cases had an estimated glomerular filtration rate decline of $\geq 10 \%$ during first-line systemic therapy.

\section{Discussion/Conclusion}

The IMDC risk model consists of 2 clinical and 4 laboratory factors that are readily available and that have been demonstrated to be associated with adverse outcomes. In this model, patients without any risk factors, in other words those who are classified into a favorable risk group, are expected to show prolonged survival. Introduction of several targeted agents in a clinical setting has resulted in more prolonged survival in this favorable risk group [6]. Indeed, in our cohort, the median PFS for first-line treatment was 29.3 months, which was much longer compared with the entire cohort (13.3 months; data not shown). Furthermore, with a median follow-up period of 46 months, the OS was not reached in our cohort (45.5 months for entire cohort; data not shown). Although our study cohort did not include favorable risk patients who were treated with first-line ICI combination therapies, long-term disease control and survival were observed even when patients were treated with single-agent therapy. On the other hand, our single-agent first-line therapy demonstrated a modest objective response rate (14.6\%), suggesting that, when systemic therapies are used to shrink tumors for symptom palliation, other treatment regimen should be considered.

Our study demonstrated that a longer duration of first-line treatment and exclusive lung metastases predicted independently a longer OS in favorable risk patients. In this study, up to $81.8 \%$ of patients received firstline systemic therapy with VEGFR-TKI and the median duration of first-line systemic therapy was 21.5 months. Based on these results, we believe favorable risk patients would rarely show primary refractory disease, and the prolonged first-line systemic therapy duration is important for successful treatment. Heng et al. [8] have reported that patients with an insufficient response to first-line systemic therapy have poor survival outcomes. Patients in their analysis were stratified into 2 groups according to the best response to first-line systemic therapy [8]. In our study, however, the duration of first-line systemic thera- py, rather than the primary refractory disease, predicted OS. Because only $2(3.6 \%)$ patients showed primary refractory disease in our study, we could not stratify the patients by primary refractory disease.

The common metastatic sites in RCC include the lung, lymph nodes, and bone [9]. Previous studies reveled that molecular profiles of metastatic sites might vary from the primary site and each other, resulting in different cancer biology [10]. These results imply that genetic heterogeneity in metastatic sites would be responsible for a mixed response to systemic therapy. In our study, favorable risk patients with lung only metastases acquired a significant survival advantage from systemic therapy. We propose 2 possible explanations for our results. The first was that favorable risk patients with lung only disease would possess favorable disease biology, especially in a favorable risk group. As can be seen from the IMDC data, the clinical outcomes of mRCC patients with lung metastases were better compared to those with metastasis to liver, brain, and pleura [9]. These results suggest systemic therapy could control lung involvement compared with other metastases. The second explanation is differences in the metastatic numbers, since it is known that oligo metastatic disease, or fewer affected organs, would result in a better prognosis [9]. It would not be surprising if favorable risk patients with lung only disease showed a better prognosis compared to patients with multiple metastatic organs.

In previous studies, we described the utility of CRP as a prognostic marker in patients with $\mathrm{mRCC}$ treated with systemic therapy [11-13]. However, in this study, the baseline CRP level did not correlate with prognosis. The mean baseline CRP value was $0.354 \mathrm{mg} / \mathrm{dL}$, which was significantly lower compared with the whole cohort $(1.904 \mathrm{mg} / \mathrm{dL}, p<0.0001$, data not shown). Based on these results, we believe that in favorable risk patients, who demonstrate a relatively lower baseline CRP level, the baseline CRP could not predict the prognosis.

Our study has several limitations that should be acknowledged. First, the selection bias of a retrospective study exists. Second, the number of patients from a single institution is limited. Third, our cohort demonstrated a longer OS despite the low objective response rate. One possible explanation would be that, our cohort included some non-clear histology cases, and first-line systemic therapy other than TKIs. We believe our analyses resulted in lower PR and PD, and a higher SD rate due to these factors. Prospective investigations of clinical and molecular features in a large number of patients with mRCC would be of great value.

Taken together, our results suggest that first-line systemic therapy for favorable risk mRCC patients with a 
single agent resulted in relatively longer PFS and OS. Additionally, these results also suggest that a longer duration of first-line treatment with systemic therapies and lung only metastases independently correlated with longer OS. These results suggest the possibility that favorable risk mRCC patients might be stratified by metastatic sites, and maximizing first-line treatment duration for these patients would prolong OS.

\section{Statement of Ethics}

Ethical approval was waived by the local Ethics Committee of Keio University in view of the retrospective nature of the study. All procedures performed in accordance with the 1964 Helsinki Declaration and its later amendments or comparable ethical standards. Because this was a retrospective cohort study, informed consent was waived. Opt-out was done on the Web site of Keio University.

\section{Conflict of Interest Statement}

R. Mizuno, MD, has received honoraria from Bristol, Ono, Merck, Takeda, and Pfizer. M. Oya, MD, has received honoraria from Bayer, Bristol, Novartis, Ono, Merck, Takeda, MSD, and Pfizer. Other authors declare that they have no conflict of interest.

\section{Funding Sources}

Supported in part by grants-in-aid from the Ministry of Education, Science, Sports, and Culture of Japan (20K09585 to R.M.)

\section{Author Contributions}

R. Mizuno carried out the design of this research, analyzed, and interpreted the data, and drafted the manuscript. K. Takamatsu, Y. Yasumizu, N. Tanaka, T. Takeda, S. Morita, K. Matsumoto, T. Kosaka, and $\mathrm{H}$. Asanuma participated in the collection of data and data analysis. S. Mikami worked on pathological assessment. M. Oya assisted in the design of this research and project development. All authors read and approved the final manuscript.

\section{Data Availability Statement}

The data that support the findings of this study are not publicly available on ethical ground but are available from the corresponding author with permission from the local Ethics Committee of Keio University.

\section{References}

1 Sternberg CN, Davis ID, Mardiak J, Szczylik C, Lee E, Wagstaff J, et al. Pazopanib in locally advanced or metastatic renal cell carcinoma: results of a randomized phase III trial. J Clin Oncol. 2010 Feb 20;28(6):1061-8.

2 Motzer RJ, Hutson TE, Tomczak P, Michaelson MD, Bukowski RM, Rixe O, et al. Sunitinib versus interferon alfa in metastatic renal-cell carcinoma. N Engl J Med. 2007 Jan 11; 356(2):115-24.

3 Motzer RJ, Tannir NM, McDermott DF, Aren Frontera O, Melichar B, Choueiri TK, et al. Nivolumab plus ipilimumab versus sunitinib in advanced renal-cell carcinoma. N Engl J Med. 2018 Apr 5;378(14):1277-90.

4 Motzer RJ, Penkov K, Haanen J, Rini B, Albiges L, Campbell MT, et al. Avelumab plus axitinib versus sunitinib for advanced renalcell carcinoma. N Engl J Med. 2019 Mar 21; 380(12):1103-15.

5 Rini BI, Plimack ER, Stus V, Gafanov R, Hawkins R, Nosov D, et al. Pembrolizumab plus axitinib versus sunitinib for advanced renal-cell carcinoma. N Engl J Med. 2019 Mar 21;380(12):1116-27.
6 Heng DY, Xie W, Regan MM, Warren MA, Golshayan AR, Sahi C, et al. Prognostic factors for overall survival in patients with metastatic renal cell carcinoma treated with vascular endothelial growth factor-targeted agents: results from a large, multicenter study. J Clin Oncol. 2009 Dec 1;27(34):5794-9.

7 Eisenhauer EA, Therasse P, Bogaerts J, Schwartz LH, Sargent D, Ford R, et al. New response evaluation criteria in solid tumours: revised RECIST guideline (version 1.1). Eur J Cancer. 2009 Jan;45(2):228-47.

8 Heng DY, Mackenzie MJ, Vaishampayan UN, Bjarnason GA, Knox JJ, Tan MH, et al. Primary anti-vascular endothelial growth factor (VEGF)-refractory metastatic renal cell carcinoma: clinical characteristics, risk factors, and subsequent therapy. Ann Oncol. 2012 Jun;23(6):1549-55.

9 Dudani S, de Velasco G, Wells JC, Gan CL, Donskov F, Porta C, et al. Evaluation of clear cell, papillary, and chromophobe renal cell carcinoma metastasis sites and association with survival. JAMA Netw Open. 2021 Jan 4; 4(1):e2021869.
10 Bianchi M, Sun M, Jeldres C, Shariat SF, Trinh QD, Briganti A, et al. Distribution of metastatic sites in renal cell carcinoma: a population-based analysis. Ann Oncol. 2012 Apr; 23(4):973-80.

11 Mizuno R, Kimura G, Fukasawa S, Ueda T, Kondo T, Hara H, et al. Angiogenic, inflammatory and immunologic markers in predicting response to sunitinib in metastatic renal cell carcinoma. Cancer Sci. 2017 Sep;108(9): 1858-63.

12 Takamatsu K, Mizuno R, Omura M, Morita S, Matsumoto K, Shinoda K, et al. Prognostic value of baseline serum $\mathrm{C}$-reactive protein level in intermediate-risk group patients with metastatic renal-cell carcinoma treated by first-line vascular endothelial growth factortargeted therapy. Clin Genitourin Cancer. 2018 Aug; 16(4):e927-e33.

13 Takamatsu K, Mizuno R, Tanaka N, Takeda T, Morita S, Matsumoto K, et al. Prognostic value of serum C-reactive protein level prior to second-line treatment in intermediate risk metastatic renal cell carcinoma patients. Int J Clin Oncol. 2019 Sep;24(9):1069-74. 